\title{
The ATLAS High Level Trigger Infrastructure, Performance and Future Developments
}

\author{
F. Winklmeier on behalf of the ATLAS Collaboration \\ CERN, PH/ADT Department, CH-1211 Geneva, Switzerland
}

\begin{abstract}
The ATLAS High Level Trigger (HLT) is a distributed real-time software system that performs the final online selection of events produced during proton-proton collisions at the Large Hadron Collider (LHC). It is designed as a two-stage event filter running on a farm of commodity PC hardware. Currently the system consists of about 850 multi-core processing nodes that will be extended incrementally following the increasing luminosity of the LHC to about 2000 nodes depending on the evolution of the processor technology. Due to the complexity and similarity of the algorithms a large fraction of the software is shared between the online and offline event reconstruction. The HLT Infrastructure serves as the interface between the two domains and provides common services for the trigger algorithms. The consequences of this design choice will be discussed and experiences from the operation of the ATLAS HLT during cosmic ray data taking and first beam in 2008 will be presented. Since the event processing time at the HLT is directly related to the number of processing nodes required, special emphasis has to be put on monitoring and improving the performance of the software. Both open-source as well as custom developed tools are used for this task and a few usecases will be shown. Finally, the implications of the prevailing industry trend towards multi- and many-core processors for the architecture of the ATLAS HLT will be discussed. The use of multi-processing and multi-threading techniques within the current system will be presented. Several approaches to make optimal use of the increasing number of cores will be investigated and the practical implications of implementing each approach in the current system with hundreds of developers and several hundred thousand lines of code will be examined.
\end{abstract}

\section{INTRODUCTION}

A TLAS [1] is a general purpose detector built for collecting data at the Large Hadron Collider (LHC) at CERN [2]. It covers a diversified physics program ranging from discovery physics to precision measurements of the Standard Model parameters and understanding the mechanism of electroweak symmetry breaking. At the design luminosity of $10^{34} \mathrm{~cm}^{-2} \mathrm{~s}^{-1}$ with a center of mass energy of $14 \mathrm{TeV}$ it will produce proton-proton collisions at a bunch crossing rate of $40 \mathrm{MHz}$. With 25 overlapping interactions per bunch crossing expected, the interaction rate of $10^{9} \mathrm{~Hz}$ must be reduced online by seven orders of magnitude in order to obtain a final event rate manageable in terms of storage space and offline processing. In a first stage the LHC is expected to deliver colliding beams in 2009 with a center of mass energy of $10 \mathrm{TeV}$ at a luminosity of $10^{31} \mathrm{~cm}^{-2} \mathrm{~s}^{-1}$.

\section{The ATLAS TRIGger}

The ATLAS trigger system achieves this rate reduction by using a multi-level architecture where the event selection deci- sion is split into three different stages of increasing complexity and processing time.

The Level-1 trigger (L1) uses algorithms implemented on custom hardware boards that process only a subset of the event data coming from the muon spectrometer and the calorimeter with coarse granularity. The output rate of the L1 trigger is designed to be approximately $75 \mathrm{kHz}$, but upgradeable to $100 \mathrm{kHz}$ with a fixed latency of $2.5 \mu$ s. For accepted events small localized Regions of Interest (RoI) in pseudo rapidity $\eta$ and azimuthal angle $\phi$ centered around the high $p_{\mathrm{T}}$ objects are identified. Each RoI contains the type and thresholds passed of the associated high $p_{\mathrm{T}}$ candidate object.

After the initial L1 selection, the event data from the various sub-detectors is held in separate memory buffers and waits for a decision to determine if it is assembled into a complete event for the final selection or discarded. This decision is taken in the Level-2 (L2) trigger that analyzes the data using fast algorithms performing an approximated reconstruction of the physics candidates. The trigger algorithms make use of the full detector data but access only the detector regions within the RoIs identified in the previous step by L1. This RoI-based access requires only about $2-4 \%$ of the event data to take the L2 decision, thus limiting the required bandwidth. The L2 output rate is about $3 \mathrm{kHz}$ with a typical event processing time of $40 \mathrm{~ms}$. The data, after being accepted by L2, are assembled into the full event and delivered to the Event Filter (EF). The algorithms employed at this stage are very similar to those used in the offline reconstruction software and make use of the fully detailed detector condition data. The increase of the reconstruction accuracy provides a decrease in the L2 accept rate to about $200 \mathrm{~Hz}$. But the use of more sophisticated algorithms increases the time to process an event, with the approximated latency of the Event Filter estimated to be about 4 seconds. Both L2 and EF form the High Level Trigger (HLT).

\section{The ATLAS High LeVel Trigger}

\section{A. High Level Trigger Hardware}

The HLT applications run on standard PC hardware mounted in one rack units (1U). One rack consists of 31 machines. Currently there are 27 racks installed that corresponds to about $35 \%$ of the final system. Over the coming months and following the LHC luminosity the system will be expanded to its final size of 79 racks. Out of this total, 17 racks will be used as L2 processors and 28 racks can be freely configured as L2 or EF nodes allowing for an optimal 
use of the available resources. The remaining nodes are used as EF processing nodes. All machines are network booted and each rack contains a local file server that is synchronized with the central file server hosting the HLT software and other files required for the running of the HLT applications. The currently installed nodes contain two CPU sockets with each one quadcore Intel Harpertown CPU running at $2.5 \mathrm{GHz}$. In addition each node has two $1 \mathrm{Gbit} / \mathrm{s}$ network interfaces and $16 \mathrm{~GB}$ of memory ( 2 GB per core). Scientific Linux CERN 5 is used as the operating system.

\section{B. High Level Trigger Software Environment}

The L2 and EF software is running inside two Dataflow applications called the L2 and EF processing unit (L2PU/EFPU). The L2PU application is able to run multiple worker threads each processing one event in parallel. The EFPU always processes one event per processing task. More details about usage of multi-threading techniques in the HLT will be discussed in section IV. However, both applications use multiple threads to handle data requests, process control, histogram publishing and other online related activities asynchronous to the event processing in the HLT algorithms. Both applications are controlled by the central run controller and follow the transitions of the ATLAS finite state machine.

The HLT software environment is schematically shown in Fig. 1. It is largely based on the offline software framework Athena [3], which itself is based on Gaudi [4], a co-development between LHCb and ATLAS. Gaudi/Athena is a component-based framework. Abstract interfaces ensure that each component can be replaced without changes in the clients. This is extremely useful for the HLT. It allows the implementation of special services with online-specific features. The HLT algorithms itself are controlled by the HLT steering [5] that is part of the HLT Core Software and schedules the execution of each algorithm. The result of the algorithm execution is then communicated to the underlying HLT framework.

In both the L2 and EF a large fraction of the offline reconstruction code is being reused. This allows for an efficient development of new algorithms and guarantees the consistency of trigger performance evaluations required for physics analysis that are mostly performed in the offline. Examples for reutilized components are the event store, the event data model, the detector description, the conditions data service and many reconstruction tools used in the EF. Further examples include services that read selected detector conditions data from the online information system and present them to the algorithms as if they would have been retrieved from the offline conditions database. This situation is schematically shown in Fig. 2. A special case is the online job configuration where the complete trigger configuration [6] is read from a database instead of executing the Python configuration scripts as in the offline.

Also shown in Fig. 1 are the three domains where the HLT software is being used: offline, integration and online. Each domain has its own specific requirements and use-cases. For the algorithm developer this should be as transparent as possible and not require any code changes when moving
Algorithms

\section{HLT Core Software}

\section{Athena / Gaudi}

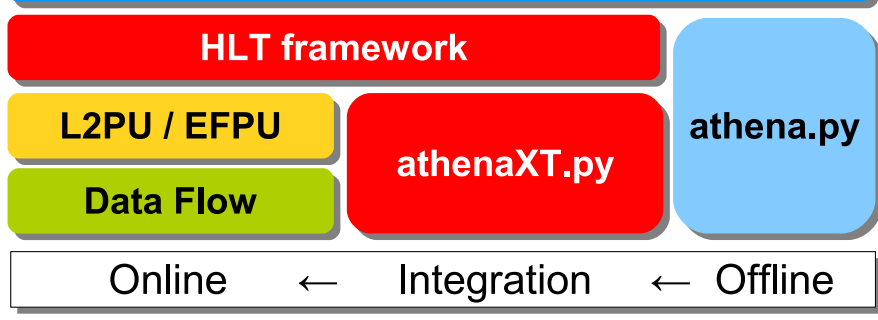

Fig. 1. Layers and domains of the ATLAS High Level Trigger software. Each layer defines abstract interfaces that ensures code stability and portability between the offline, integration and online domain.

from one domain to the other. The HLT framework contains special implementations of some of the offline services (e.g. for histogram publishing, messaging, data requests, etc.) that are used when running the HLT online or in the integration environment. The latter is implemented by two special command line applications, athenaMT for L2 and athenaPT for the EF. They are written in Python and emulate the environment of a L2PU and EFPU, respectively. They share a common code base and differences arise only in the way the HLT event selection software accesses raw data. To simulate the data access via network requests for L2, athenaMT loads the raw data fragments for each event into memory and provides them on algorithm request to the event selection software. In a similar way athenaPT provides the fully built event. Both emulators allow in an interactive mode to cycle through the ATLAS finite state machine. This is invaluable since problems can already be found before running the code online. Command line options for the emulators have been implemented as close as possible to the ones available within the offline athena executable. Developers familiar with the offline environment can thus easily get acquainted with the HLT development and need not to be familiar with detailed technical aspects of the Dataflow software. In addition to providing a transparent environment, the HLT framework also shields the developers from changes in the Dataflow software itself.

\section{Release building and validation}

The software packages belonging to the HLT infrastructure form the so called AtlasHLT software project. It depends on most of the offline software projects. Due to its nature as an interface package between the offline and online software it is the only software project that can depend on both the offline and online (tdaq) software. Fig. 3 shows the dependency graph of the AtlasHLT project. It is evident that releases have to be coordinated both with the online as well as the offline release coordinators to allow for a common deployment schedule of new releases. This is especially important if there are changes to common external packages (e.g. boost, LCG, ROOT, ...) 


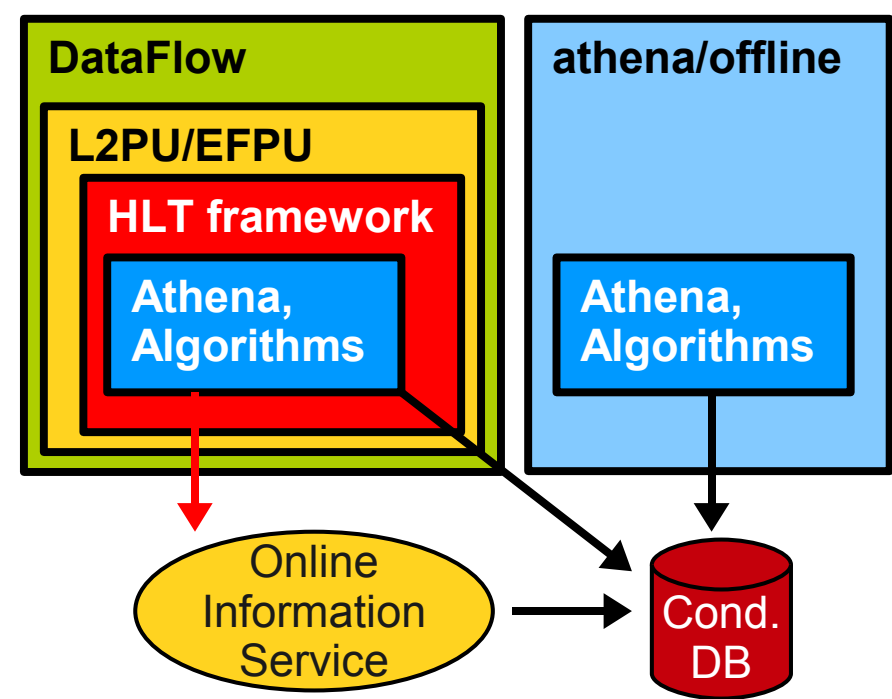

Fig. 2. Schematic view of the online and offline HLT environment where parts of the conditions data used by the algorithms is being read from the online information service and others are read directly from the conditions database. This is necessary since some detector conditions are not available in the conditions database at the time of data taking and are only recorded therein during the course of the run.

that have to be synchronized across all projects. To allow for fast patches to existing releases the AtlasP1HLT project can be used to patch any existing package in the offline and AtlasHLT projects. Of course, it has to be taken care that no public interface changes are introduced in such a patch.

The same tools and procedures as in the offline [7] are used for the building and validation of the AtlasHLT release. Developers collect new versions of their packages with the ATLAS TagCollector [8] and a new release is built every night. Automatic tests are run after each build and common tools have been developed together with the offline trigger validation group to ease the analysis and display of the test results [9]. The automatic nightly tests consist of both functional as well as regression tests. This includes tests of the HLT infrastructure, stopping and starting of runs, up to small Dataflow setups where the L2 and EF applications are running on the same node and a small number of events are played through the system. All these test produce histograms and $\log$ files that are compared to references and automatically checked for error and warning messages. The same set of tests is used to decide whether a new AtlasP1HLT release is ready to be deployed for data taking.

\section{Performance Monitoring}

The HLT poses strict performance requirements on execution times and memory usage of the algorithms. These requirements are usually much stricter than in the offline, sometimes even contradictory. A few examples are given in the following.

All initializations (e.g. data structures, loading of shared libraries or conditions data) have to be done before the first event. Otherwise the event processing time at the start of a new run will exceed the Dataflow timeouts and the event will be discarded into a special debug stream. On the other

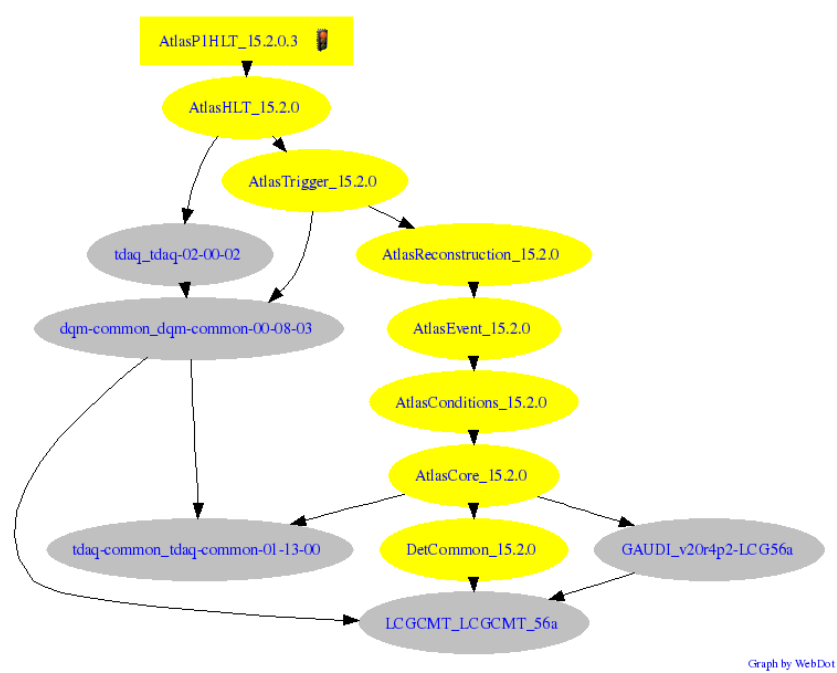

Fig. 3. Dependency tree of the full ATLAS software including the AtlasHLT infrastructure project and the AtlasP1HLT patching project. Also shown are the online projects (tdaq, tdaq-common, dqm-common) as well as the major external projects LCG and Gaudi. Note that both tdaq and offline projects directly depend on LCG and releases have to be synchronized in case of version changes in these externals.

side, this is not a concern for the offline software where most initializations are done on-demand whenever a component is used for the first time. In order to identify code that does timeconsuming initializations on the first event, a special tool based on valgrind's [10] callgrind tool was developed. By using the monitoring hooks provided within the Athena framework it is able to dump execution profiles for the first and Nth event separately. A python script analyzes the two profiles and shows the differences sorted by number of function calls or instruction costs.

Memory usage is the most important system resource to monitor for both the HLT and offline reconstruction software. For both applications the hard limit is 2 GB of real memory imposed by the available memory of the HLT nodes and the Grid production nodes, respectively. For the HLT there are very strict limits on the size of possible memory leaks. The job lifetime of a reconstruction job is typically on the order of ten thousand events whereas an HLT application needs to be able to process millions of events without significant increase in memory usage. For example, in L2 running at $25 \mathrm{~Hz}$, a memory leak of $10 \mathrm{kB}$ per event will grow to about $900 \mathrm{MB}$ in just one hour. To avoid these surprises when running the algorithms online, a continuous monitoring of the memory usage has been put in place. Fig. 4 shows the memory usage of a typical EF application running over $1000 t \bar{t}$ events. It can be seen that after the initial increase of memory usage it stabilizes and no further growth is visible. These plots are created for a large number of different job configurations automatically every night using the ATLAS Run Time Tester [11]. In addition to the monitoring of the memory usage, open-source and custom leak checkers are used to find memory leaks in the code. Due to the very large code base it is important that these tests execute as many of the code paths as possible. Therefore events resulting in high detector occupancy, like the aforementioned $t \bar{t}$ or black hole events, 


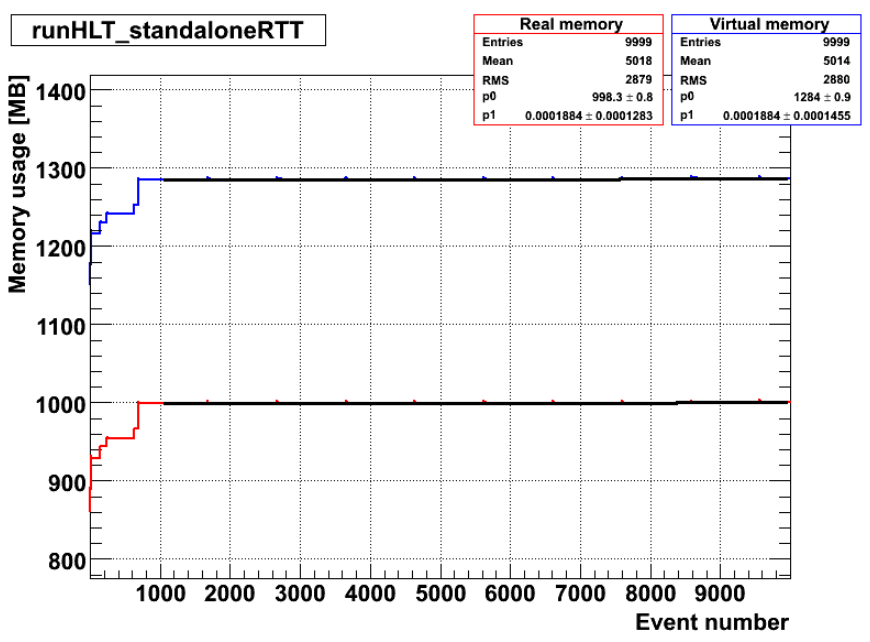

Fig. 4. Real (top, red) and virtual memory (bottom, blue) usage of a typical $\mathrm{EF}$ application running over $1000 t \bar{t}$ events. After the initial increase the memory usage stabilizes indicating very small per-event memory leaks.

have to be used.

\section{E. Operational Experience}

The HLT framework has been used for more than five years. It was first deployed in a setup with detector components for the ATLAS test beam in 2004 [12]. It was used for many data taking periods with cosmics in 2007 and 2008, including the combined ATLAS cosmic runs, and it was configured in pass through mode for first LHC events in September 2008. These data taking periods exercised all core software components from algorithm configuration with the trigger database to event streaming initiated by algorithm decisions. In the cosmic data taking period starting on September 13, 2008, 216 million events were handled by the HLT framework with a data volume of 453 TB in 400000 files (Fig. 5). About 3\% of the events that caused data taking problems, mainly due to corrupted raw data fragments or event timeouts, were written to the debug stream.

While cosmic data taking provides an invaluable operational experience with an integrated detector setup, the Trigger/DAQ system and the HLT framework are not pushed to its performance and throughput limits. These limits were explored in several so called "Trigger/DAQ Technical Runs". For these periods the read-out system was preloaded with simulated data. The data fragments from different sub-detectors were assigned in a realistic way to the read-out drivers and the full HLT event selection menu for a luminosity of $10^{31} \mathrm{~cm}^{-2} \mathrm{~s}^{-1}$ was configured. All available HLT processors were attributed to the L2 farm running $2880 \mathrm{~L} 2 \mathrm{PU}$ instances that corresponds to roughly $70 \%$ of the final system. In a seven hour lasting run, an input rate of $60 \mathrm{kHz}$ could be sustained (Fig. 6). This value corresponds to about $80 \%$ of the design value. In the course of this test sharp drop in the rate were observed that could be correlated to system cron jobs.

\section{Parallelism and Multi-Core CPUs}

Typical high energy physics applications, i.e. the HLT applications, fall in the class of "embarrassingly parallel" problems

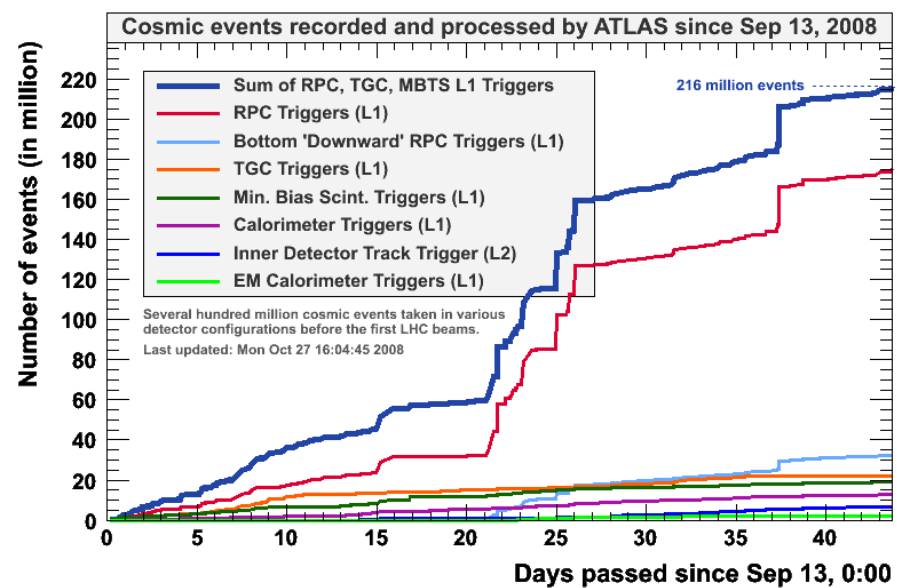

Fig. 5. Number of cosmic events recorded in the cosmic run starting on September 13, 2008 after the LHC incident. 216 million events were processed and streamed by the HLT with a data volume of $453 \mathrm{~TB}$.

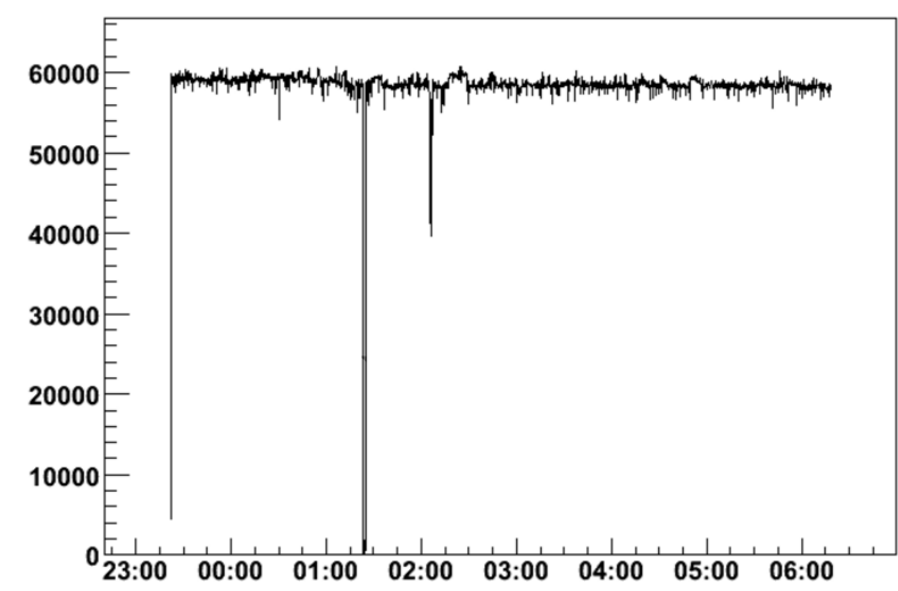

Fig. 6. L2 input rate in $\mathrm{Hz}$ during a test run lasting seven hours. Simulated data were preloaded on the read-out system and pushed through a setup with $2880 \mathrm{~L} 2$ processing units. This corresponds to about $70 \%$ of the final system. The sharp drops in rate are caused by system cron jobs.

if the event-level parallelism is exploited. Since two events are independent of each other they can be processed in parallel in any given order. No synchronization is needed between the different processing tasks. To achieve the parallelism one can either use multi-threading or multi-processing techniques. For the first, an application contains several worker threads of which each thread processes one event. For the latter, several processes are used to process events in parallel. Which of the approaches to choose depends mostly on additional boundary conditions of the applications at hand.

The original design of the ATLAS HLT applications as described in the 2003 technical design report [13] exploits event parallelism to achieve the required event throughput. In L2 concurrent worker threads execute the algorithm code in parallel on each processor, while concurrent processing tasks work in parallel on the full event in the EF. By the time of first data taking, a typical HLT processor was believed to run on a dual-socket, single-core CPU machine running at a clock speed of about $8 \mathrm{GHz}$. It was foreseen to start on each L2 processor one instance of an L2PU application with up to three 


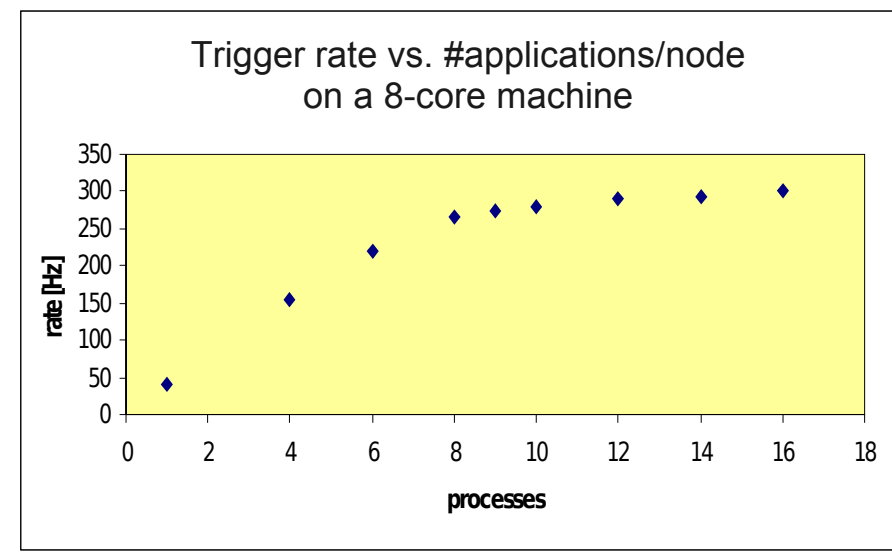

Fig. 7. Scaling of the event throughput rate with the number of HLT processes on a dual CPU quad-core machine. There is almost linear scaling up to eight processes, i.e. one process per CPU core. Running more selection processes than available CPU cores does not significantly increase the event rate.

worker threads and two instances of EFPUs per EF processor. Early on it became clear that it is very difficult to maintain a large code base thread-safe and thread-efficient in an open developer community. It requires careful tuning and expert knowledge in multi-threaded programming, which only very few physicists have. In addition the amount of code re-use from offline projects is very limited, since this code was never developed for a multi-threaded environment. While in the beginning certain selection chains could run multi-threaded, it proved to be very difficult to maintain this capability over release cycles. Dependencies on external software and system libraries made this task even harder. E.g. it was found that the allocation scheme of the STL at that time did not allow for efficient execution of multiple threads [14]. Finally, there is a large impact on the turn-around time for code improvements and bug fixes if each change has to be accompanied with a time-consuming validation and performance study. As a consequence the L2PU is now configured to run with one worker thread but it still uses the multi-threaded infrastructure for data retrieval, monitoring and run control. The required throughput is achieved by starting one L2PU instance per CPU core equivalent to the original design of the EF applications. Fig. 7 shows the scaling of the event rate with the number of applications on an eight-core machine. We can see linear scaling up to the point where more than one application is started per core. No code changes are required on the algorithm side and each processor is independent of the others. I.e. if one application crashes only the currently processed event has to be recovered, whereas in the multi-threaded version one usually has to recover all events that were processed at that time in the worker threads.

As explained above, the multi-threaded approach to eventlevel parallelism has been abandoned in the ATLAS HLT in favor of multiple processes per node, i.e. one L2PU or EFPU application per core. As a consequence the usage of system resources (e.g. memory, sockets, number of network connections and controlled applications, etc.) is being multiplied. Over the past decade Moore's doubling of transistors was always accompanied by a similar increase in clock speed therefore providing a "free lunch" for application developers. This era has clearly come to an end with the introduction of multiand many-core CPUs and stagnating clock speeds. Although, each individual CPU core is still providing an increase in performance from one generation to the next, the bulk of the additional transistors is being used for additional cores on the same chip. It is important to ensure that our applications will make optimal use of the increased performance in multi-core CPUs if we want to continue profiting of Moore's law. This is particularly true because we will definitely see the first manycore CPUs with more than 24 hardware threads per socket during the next decade of LHC operations [15].

There are several possible solutions to the problem of resource multiplication. Multi-threading and multi-processing are on different ends of the resource sharing scale. In multithreaded applications all resources are shared by default, which of course is the reason for its notoriously difficult usage in high energy physics applications. On the other end, multiple processes do not share any resources besides what is provided through the operating system, e.g. shared libraries. Ideally the Athena framework itself would provide centrally managed shared memory segments where it would store immutable data objects and provide access to the worker processes. Studies in this direction have been done, but it requires significant code changes in the framework and possibly the algorithms itself. Two less invasive solutions to the problem are described in the following.

Recently, there have been attempts to exploit the copyon-write features of the Linux kernel in the ATLAS offline reconstruction software [16]. An already initialized process is being forked into multiple processes just before the processing of the first event starts. By default the kernel shares all the memory pages between the processes until one process modifies one of its pages. Only then is the memory page being copied and becomes unshared. Adopting this model for the online operations of the HLT is far from trivial since, unlike in the offline, HLT applications need to be known to and communicate with other parts of the DAQ system. Therefore simply duplicating existing processes will not work. Another drawback of this approach is that pages that became unshared will never be shared again, although all processes might have written the same data into the pages and they are in fact identical. Advantage of this solution is that code changes are limited on the framework side and do not affect the algorithms.

Based on the same idea of sharing identical pages between processes is the new kernel module ksm from RedHat [17]. After registering memory pages with $\mathrm{ksm}$ it scans all pages and merges identical ones. The usual copy-on-write mechanism takes care of the un-sharing once a processes tries to modify a shared page. By using periodic scans of all pages one can even merge pages again that have been modified by the processes but are still identical. Initial tests have been done with the HLT applications by running eight identical processes with $\mathrm{ksm}$ enabled. Only a small additional amount of shared pages were observed compared to running one process. This is believed to be due to non-identical memory layouts of the processes. Further work is ongoing to understand and improve the situation. Assuming acceptable sharing ratios could be 
achieved with $\mathrm{ksm}$, it would solve the problem of sharing memory between processes in a most elegant way without any code changes on the HLT side. Of course, this does not solve any of the other resource multiplication issues.

\section{CONCLUSIONS}

The HLT framework based on the Athena offline framework has been successfully used in many different data taking periods and provides the required throughput and performance. It realizes a homogeneous software and development environment from the HLT to offline and allows to benefit from a big developer community and software base. Close collaboration with the offline developers made it possible to reach the execution speed, robustness and memory leak targets necessary for trigger operations. The ability to develop, test and optimize trigger algorithms in an offline-type environment is used successfully to establish the ATLAS trigger menu. The prevailing industry trend toward multi- and many-core CPUs poses new challenges for the optimal resource usage in the HLT. Further framework developments to support these new hardware platforms are needed. Many of these issues are shared with the offline reconstruction and can profit from common solutions.

\section{REFERENCES}

[1] G. Aad et al., "The ATLAS Experiment at the CERN Large Hadron Collider," JINST, vol. 3, p. S08003, 2008.

[2] L. Evans and P. Bryant, "LHC Machine," JINST, vol. 3, p. S08001, 2008.

[3] P. Calafiura, W. Lavrijsen, C. Leggett, M. Marino, and D. Quarrie, "The athena control framework in production, new developments and lessons learned."

[4] G. Barrand et al., "GAUDI - A software architecture and framework for building HEP data processing applications," Comput. Phys. Commun., vol. 140, pp. 45-55, 2001. [Online]. Available: http://projgaudi.web.cern.ch

[5] A. Hoecker et al., "Overview of the High Level Trigger Steering and Selection for the ATLAS experiment at the LHC," IEEE Trans. Nucl. Sci., vol. 55, pp. 165-171, 2008.

[6] D. Berge et al., "The configuration system of the ATLAS Trigger," IEEE Trans. Nucl. Sci., vol. 55, pp. 392-398, 2008.

[7] F. Luehring, "Organization, Management, and Documentation of ATLAS Offline Software Releases," CHEP 2009, contribution 248, Prague, March 21-27, 2009.

[8] E. Obreshkov et al., "Organization and management of ATLAS software releases," Nucl. Instrum. Meth., vol. A584, pp. 244-251, 2008.

[9] E. Ferreira De Lima et al., "Software Validation Infrastructure for the Atlas High-Level Trigger," Proceedings of Science, 2008, poS(ACAT08)084

[10] N. Nethercote and J. Seward, "Valgrind: A Framework for Heavyweight Dynamic Binary Instrumentation." Proceedings of ACM SIGPLAN 2007 Conference on Programming Language Design and Implementation (PLDI), 2007. [Online]. Available: http://www.valgrind.org

[11] S. Brinick, "The ATLAS RunTimeTester software," CHEP 2009, contribution 140, Prague, March 21-27, 2009.

[12] A. dos Anjos et al., "Deployment of the ATLAS high-level trigger," IEEE Trans. Nucl. Sci., vol. 53, pp. 2144-2149, 2006.

[13] P. Jenni, M. Nessi, M. Nordberg, and K. Smith, ATLAS high-level trigger, data-acquisition and controls: Technical Design Report, ser. Technical Design Report ATLAS. Geneva: CERN, 2003.

[14] W. Wiedenmann, "The ATLAS online High Level Trigger framework: experience reusing offline software components in the ATLAS trigger," CERN, Geneva, Tech. Rep. ATL-DAQ-PROC-2009-011. ATL-COMDAQ-2009-039, May 2009.

[15] R. M. Ramanathan and V. Thomas, "Platform 2015: Intel Processor and Platform Evolution for the Next Decade," 2005, Intel Corporation.

[16] S. Binet et al., "Harnessing multicores: strategies and implementations in ATLAS," CHEP 2009, contribution 244, Prague, March 21-27, 2009.
[17] I. Eidus, "ksm - dynamic page sharing driver for linux," 2008, RedHat. [Online]. Available: http://lwn.net/Articles/306642 